\title{
Imaging of Cervicothoracic Junction Trauma [Corrigendum]
}

Wongwaisayawan S, Suwannanon R, Kaewlai R. Reports in Medical Imaging. 2013;6:11-27.

On page 18, Figure 5 legend is incorrect. The correct legend is:

Figure 5 (A-C) Bilateral facet fractures/dislocations.

Notes: Sagittal-reformatted computed tomography images from right to left demonstrate a fracture of the right $\mathrm{C} 7$ superior articular process (short arrows), perched right $\mathrm{C6} /$ 7 facet and grade II/III anterolisthesis of $\mathrm{C} 6$ on $\mathrm{C} 7$, and dislocation of the left $\mathrm{C} 6 / 7$ facet (long arrow).

On page 22, Figure 9 legend is incorrect. The correct legend is:

Figure 9 (A-C) Unilateral facet dislocation. An axial computed tomography image (A) reveals a reversed ham- burger-bun sign (short arrow) of the left C6/7 facet. Sagittal-reformatted computed tomography images (B and C) show grade I anterolisthesis of C6 on C7 and a jumped $\mathrm{C} 6 / 7$ left facet (long arrow).

On page 24, Figure 11, the legend is incorrect. The correct legend is:

Figure 11 (A-D) Blunt vertebral artery injury. An axial computed tomography image (A) shows a severely comminuted fracture (arrow) of the left C6 articular pillar, pedicle, lamina, and transverse process. Axial (B) and curved reformat of the left vertebral artery $(\mathbf{C})$ postcontrast computed tomography reveal absence of flow in the left vertebral artery at the level of fracture, above and below it (black arrows). Note and injury depicted on diagram $\mathbf{D}$ and a normal right vertebral artery in $\mathbf{B}$ (arrowhead).

The authors apologize for these errors. 